\title{
ABSOLUTELY SUMMING AND DOMINATED OPERATORS ON SPACES OF VECTOR-VALUED CONTINUOUS FUNCTIONS
}

\author{
BY
}

CHARLES SWARTZ

\begin{abstract}
A. Pietsch has shown that the class of dominated linear operators on $C(S)$ coincides with the class of absolutely summing operators. If the space $C(S)$ is replaced by $C_{X}(S)$, where $X$ is a Banach space, this is no longer the case. However, any absolutely summing operator is always dominated, and the classes of operators coincide exactly when $X$ is finite dimensional. A characterization of absolutely summing operators on $C_{X}(S)$ is given.
\end{abstract}

In this paper we consider absolutely summing and dominated linear operators on spaces of vector-valued continuous functions. In the case where the domain of the operators is $C(S)$, these two classes of operators coincide $[6,2.3 .4]$, but in the first section we show that this is not the case when vector-valued functions are considered. In the second section we give a characterization of absolutely summing maps on spaces of vector-valued continuous functions.

Throughout this paper $X, Y$ will denote Banach spaces, and $S$ will denote a compact Hausdorff space with the $\sigma$-algebra $\mathcal{B}$ of Borel sets. The space of all continuous functions $f: S \rightarrow X$ will be denoted by $C_{X}(S)$, and it will be assumed that $C_{X}(S)$ is equipped with the sup norm, $\|f\|=\sup \{\|f(t)\|: t \in S\}$. In case $X$ is either the real or complex numbers, we abbreviate $C_{X}(S)$ to $C(S)$.

A continuous linear map $T: C_{X}(S) \rightarrow Y$ is said to be dominated if there is a positive regular measure $\nu$ on $\mathcal{B}$ such that

$$
\|T f\| \leqq \int_{S}\|f(t)\| d \nu(t) \quad \text { for } f \in C_{X}(S)
$$

[2, III.19.3].

A continuous linear map $T: X \rightarrow Y$ is said to be absolutely summing if $T$ carries weakly unconditionally convergent series (w.u.c. series) into absolutely convergent series ([6, Chapter 2]; [4, Anhang]). (Here we are using the terminology of Pełczyński [1]; a series $\sum x_{n}$ in $X$ is said to be w.u.c. if $\sum\left|\left\langle x^{\prime}, x_{n}\right\rangle\right|<\infty$ for each $x^{\prime} \in X^{\prime}$.) We denote the space of all absolutely summing operators from $X$ into $Y$ by $A S(X, Y)$.

Theorem 2.3.4 of [6] establishes the relationship between the dominated and absolutely summing maps defined on $C(S)$. Indeed, from this result it follows that a continuous linear operator $T: C(S) \rightarrow Y$ is dominated iff $T$ is absolutely

Received by the editors August 3, 1971 and, in revised form, February 25, 1972.

AMS (MOS) subject classifications (1970). Primary 46E40, 46G10, 47B99.

Key words and phrases. Dominated operator, absolutely summing operator, vector measure of bounded variation.

Copyright $\oplus 1973$, American Mathematical Society 
summing. In $\S 1$ we show that this is not the case for operators defined on spaces of type $C_{X}(S)$.

I would like to thank the referee for his useful comments. Part (ii) of Theorems 7, 8, 11 and 12 are due to his remarks. Example 1 which gives a negative answer to a problem posed in the original version of the paper is also due to the referee.

1. Dominated and absolutely summing maps. We first show that any absolutely summing operator is dominated. For this we require several preliminary results.

Lemma 1. Let $X$ be a B-space. $A$ series $\sum x_{n}$ is w.u.c. in $X$ iff the set $A=\left\{\sum_{n \in \sigma} x_{n}: \sigma \in F(N)\right\}$ is bounded, where $F(N)$ denotes the family of all finite subsets of the positive integers $N$.

Proof. If $\sum x_{n}$ is w.u.c., then for any $\sigma \in F(N)$ and $x^{\prime} \in X^{\prime},\left|\left\langle x^{\prime}, \sum_{\sigma} x_{n}\right\rangle\right|$ $\leqq \sum_{1}^{\infty}\left|\left\langle x^{\prime}, x_{n}\right\rangle\right|\langle\infty$ so $A$ is bounded by the uniform boundedness principle.

If $A$ is bounded, there exists $M>0$ such that $\left\|\sum_{\sigma} x_{n}\right\| \leqq M$ for all $\sigma$. If $x^{\prime} \in X^{\prime}$, then $\left|\left\langle x^{\prime}, \sum_{\sigma} x_{n}\right\rangle\right| \leqq M\left\|x^{\prime}\right\|$ so that $\sum_{1}^{\infty}\left|\left\langle x^{\prime}, x_{n}\right\rangle\right| \leqq 4 M\left\|x^{\prime}\right\|[6,1.1 .2]$ and $\sum x_{n}$ is w.u.c.

Lemma 2. If $\sum \phi_{n}$ is w.u.c. in $C(S)$, then $\sum\left|\phi_{n}\right|$ is w.u.c. in $C(S)$.

Proof. By Lemma 1 , there exists $M>0$ such that $\left\|\sum_{\sigma} \phi_{n}\right\| \leqq M$ for $\sigma$ $\in F(N)$. Thus for each $t \in S,\left|\sum_{\sigma} \phi_{n}(t)\right| \leqq M$ for $\sigma \in F(N)$, and $\sum_{\sigma}\left|\phi_{n}(t)\right|$ $\leqq 4 M$ for $\sigma \in F(N)[6,1.1 .2]$. Hence $\left\|\sum_{\sigma}\left|\phi_{n}\right|\right\| \leqq 4 M$ and $\sum\left|\phi_{n}\right|$ is w.u.c. by Lemma 1 .

We denote by $\operatorname{rcabv}\left(\mathcal{B}, X^{\prime}\right)$ the set of all regular, countably additive, $X^{\prime}$-valued measures $\mu$ on $\mathcal{B}$ of finite variation equipped with the norm $\|\mu\|=v(\mu)$. (Here $v(\mu)$ denotes the variation of $\mu$ over $S$ [3, III.1.4].) The dual of $C_{X}(S)$ and $\operatorname{rcabv}\left(\mathcal{B}, X^{\prime}\right)$ are isometrically isomorphic under the pairing $\langle T, f\rangle$ $=\int_{S} f(t) d \mu(t), f \in C_{X}(S)$, where $T \in C_{X}(S)^{\prime}$ and $\mu \in \operatorname{rcabv}\left(\mathcal{B}, X^{\prime}\right)$ [5, p. 735].

Lemma 3. Let $\sum \phi_{n}$ be w.u.c. in $C(S)$ and $\left\{x_{n}\right\} \subseteq X$ be such that $\left\|x_{n}\right\| \leqq 1$. Then $\sum \phi_{n} x_{n}$ is w.u.c. in $C_{X}(S)$.

Proof. Let $\nu \in \operatorname{rcabv}\left(\mathcal{B}, X^{\prime}\right)\left(=C_{X}(S)^{\prime}\right)$. For $\sigma \in F(N)$,

$$
\left\langle\nu, \sum_{\sigma} \phi_{n} x_{n}\right\rangle=\sum_{\sigma}\left\langle\nu, \phi_{n} x_{n}\right\rangle=\sum_{\sigma} \int_{S} \phi_{n} d \nu x_{n}
$$

so that by Lemma 2 ,

$$
\begin{aligned}
\left|\left\langle\nu, \sum_{\sigma} \phi_{n} x_{n}\right\rangle\right| & \leqq \sum_{\sigma} \int_{S}\left|\phi_{n}\right| d v\left(v x_{n}\right) \leqq \int_{S}\left(\sum_{\sigma}\left|\phi_{n}\right|\right) d v(\nu) \\
& \leqq \sup \left\{\left\|\sum_{\sigma}\left|\phi_{n}\right|\right\|: \sigma \in F(N)\right\} v(\nu)(S)<\infty
\end{aligned}
$$

Hence $\left\{\sum_{\sigma} \phi_{n} x_{n}: \sigma \in F(N)\right\}$ is weak-bounded, and therefore norm-bounded. By Lemma $1, \sum \phi_{n} x_{n}$ is w.u.c. 
For our Theorem we recall the following results of Dinculeanu [2]. If $T: C_{X}(S) \rightarrow Y$ is linear and continuous, then there is a unique linear, continuous map $T^{\#}: C(S) \rightarrow L(X, Y)$ such that $T(\phi x)=T^{\#} \phi(x)$ for $\phi \in C(S), x \in X[2$, III.19.2]. (Here $L(X, Y)$ denotes the bounded linear operators from $X$ into $Y$.) Moreover, $T$ is dominated iff $T^{\#}$ is dominated [2, III.19.3].

Theorem 4. If $T: C_{X}(S) \rightarrow Y$ is absolutely summing, then $T^{\#}$ is absolutely summing.

Proof. Let $\sum \phi_{n}$ be w.u.c. in $C(S)$. Let $\varepsilon>0$, and for each $n$ pick $x_{n} \in X$ such that $\left\|x_{n}\right\| \leqq 1$ and $\left\|T^{\#} \phi_{n}\right\| \leqq\left\|T^{\#} \phi_{n}\left(x_{n}\right)\right\|+\varepsilon / 2^{n}$. Now by Lemma $3, \sum \phi_{n} x_{n}$ is w.u.c. in $C_{X}(S)$ so $\sum\left\|T\left(\phi_{n} x_{n}\right)\right\|=\sum\left\|T^{\#} \phi_{n}\left(x_{n}\right)\right\|<\infty$. Hence $\sum\left\|T^{\#} \phi_{n}\right\|$ $<\infty$, and $T^{\#}$ is absolutely summing.

Corollary 5. If $T: C_{X}(S) \rightarrow Y$ is absolutely summing, then $T$ is dominated.

Proof. By the Theorem $T^{\#}$ is absolutely summing, and thus $T^{\#}$ is dominated [6, 2.3.4]. Hence $T$ is dominated [2, III.19.3, Theorem 4].

It follows fairly readily from Pietsch's result [6, 2.3.4] that any dominated operator $T: C_{X}(S) \rightarrow Y$ is absolutely summing when $X$ is finite dimensional. For assume that $X$ is $n$-dimensional and, for convenience, assume that $X$ is a real vector space. (That is, $X=R^{n}$.) Denote by $\delta^{j}$ the vector in $X$ with 1 in the $j$ th coordinate and 0 in the other coordinates. If $T: C_{X}(S) \rightarrow Y$ is linear and bounded, then $T$ induces $n$ linear operators $T_{j}: C(S) \rightarrow Y$ defined by $T_{j}(\phi)$ $=T\left(\phi \delta^{j}\right), \phi \in C(S)$.

We then have $T f=\sum_{j=1}^{n} T_{j} f_{j}$ for $f=\left(f_{1}, \ldots, f_{n}\right)$ in $C_{X}(S)$, and $T_{j}(\phi)=T\left(\phi \delta^{j}\right)$ for $\phi \in C(S)$. From these relationships, it is easy to see that $T$ is dominated iff each $T_{j}$ is dominated iff each $T_{j}$ is absolutely summing [6,2.3.4] iff $T$ is absolutely summing.

We show that the property above actually characterizes finite dimensional spaces.

Theorem 6. If the B-space $X$ is such that any dominated linear operator $T: C_{X}(S) \rightarrow X$ is absolutely summing, then $X$ is finite dimensional.

Proof. Fix $t \in S$. Define $T: C_{X}(S) \rightarrow X$ by $T f=f(t), f \in C_{X}(S)$. Then $T$ is dominated since $\|T f\|=\int_{S}\|f(s)\| d \delta_{t}(s)$, where $\delta_{t}$ is the Dirac measure concentrated at $t$. By hypothesis $T$ is absolute summing.

Assume now that $\sum x_{n}$ is w.u.c. in $X$ and pick $\phi \in C(S)$ such that $\phi(t)=1$. Then $\sum \phi x_{n}$ is w.u.c. in $C_{X}(S)$ by Lemma 1 . Thus $\sum T\left(\phi x_{n}\right)=\sum x_{n}$ is absolutely summable. Hence by the generalized Dvoretzky-Rogers theorem of Pietsch [9, Theorem 8], $X$ is finite dimensional.

From the proof above we see that if $X$ is any infinite dimensional $B$-space, there is a dominated linear operator $T: C_{X}(S) \rightarrow X$ which is not absolutely summing. That is, the converse of Corollary 5 is not valid. 
2. Characterization of absolutely summing maps. In this section we give a characterization of absolutely summing operators on $C_{X}(S)$. Recall that a bounded linear operator $T: C_{X}(S) \rightarrow Y$ has a representation

$$
T f=\int_{S} f(t) d m(t), \quad f \in C_{X}(S),
$$

where $m: \mathcal{B} \rightarrow L\left(X, Y^{\prime \prime}\right)$ is a finitely additive measure of bounded semivariation [6, III.19.5]. (Here $L\left(X, Y^{\prime \prime}\right)$ denotes all the bounded linear operators from $X$ into $Y^{\prime \prime}$ equipped with the usual operator norm.) We refer to the measure $m$ as the representing measure of $T$, and we give a characterization of absolutely summing operators in terms of its representing measure. We also give a characterization of absolutely summing operators in terms of the operator $T^{\#}$.

Theorem 7. Let $T: C_{X}(S) \rightarrow Y$ be absolutely summing. Then:

(i) For each $B \in \mathcal{B}$ the map $m(B): X \rightarrow Y$ is absolutely summing.

(ii) For each $\phi \in C(S)$ the map $T^{\#} \phi: X \rightarrow Y$ is absolutely summing.

Proof. For (i) recall we have the formula

$$
m(B) x=T^{\prime \prime}\left(C_{B} x\right), \quad x \in X,
$$

for the representing measure $m$ (see [6, III.19.5]; here $C_{B}$ denotes the characteristic function of $B$ and $C_{B} x$ denotes the continuous linear functional defined on $\operatorname{rcabv}\left(\mathcal{B}, X^{\prime}\right)$ by $\left.\left\langle C_{B} x, \nu\right\rangle=\langle\nu(B), x\rangle\right)$.

If $\sum x_{n}$ is w.u.c. in $X$, then $\sum C_{B} x_{n}$ is also w.u.c. in $C_{X}(S)^{\prime \prime}$ by Lemma 1. Since $T^{\prime \prime}$ is absolutely summing [8, Satz 15], formula (1) shows $m(B)$ is absolutely summing so that (i) follows. For $\phi \in C(S), \sum \phi x_{n}$ is w.u.c. in $C_{X}(S)$ by Lemma 1, so $T^{\#} \phi\left(x_{n}\right)$ is absolutely summable and (ii) follows.

If $T: C_{X}(S) \rightarrow Y$ is absolutely summing, then $T$ is dominated (Corollary 5), and the measure $m$ therefore has bounded variation [6, III.19.3]. We show that it also has bounded variation with respect to the absolutely summing norm [7, §7]; [4, Anhang] and the operator $T^{\#}$ is absolutely summing from $C(S)$ into $A \delta(X, Y)$. For this we recall some facts pertaining to the absolutely summing norm.

If $E$ is a normed linear space, then $l_{w}(E)$ [4, Anhang 2.1] denotes the vector space of all sequences $\left\{x_{n}\right\}$ such that $\sum x_{n}$ is w.u.c. (weakly summable in the terminology of Pietsch [6]). (Here addition and scalar multiplication are coordinatewise as in $[6,1.2 .2]$.) The vector space $l_{w}(E)$ has a natural norm, called the $\varepsilon$ norm, defined on it via $\varepsilon\left[x_{n}\right]=\sup \left\{\sum\left|\left\langle x^{\prime}, x_{n}\right\rangle\right|: x^{\prime} \in X^{\prime},\left\|x^{\prime}\right\| \leqq 1\right\}([6,1.2 .3]$; [4, Anhang 2.1]). We denote by $l_{s}(E)$ the linear subspace of $l_{w}(E)$ consisting of all sequences $\left\{x_{n}\right\}$ such that the series $\sum x_{n}$ is unconditionally convergent and assume that $l_{s}(E)$ is equipped with the $\varepsilon$-norm from $l_{w}(E)$. Finally, we denote by $l_{a}(E)$ the vector space of all sequences $\left\{x_{n}\right\}$ such that the series $\sum x_{n}$ is absolutely convergent and we assume that $l_{a}(E)$ is equipped with the $\pi$-norm defined by $\pi\left[x_{n}\right]=\sum\left\|x_{n}\right\|([4$, Anhang 2.4]; [6, 1.1.4]). 
A linear, continuous operator $T: X \rightarrow Y$ induces a linear map $T^{*}: l_{w}(X)$ $\rightarrow l_{w}(Y)$ by $T^{*}\left(\left[x_{n}\right]\right)=\left[T x_{n}\right]$, and $T$ is absolutely summing iff $T^{*}\left(l_{w}(X)\right)$ $\subseteq l_{a}(Y)$ iff $T^{*}\left(l_{s}(X)\right) \subseteq l_{a}(Y)$ ([4, Anhang 3]; [6, 2.1]). The absolutely summing norm or $\pi$-norm of an absolutely summing operator $T: X \rightarrow Y$ is defined to be the operator norm of $T^{*}: l_{w}(X) \rightarrow l_{a}(Y)$ (or $T^{*}: l_{s}(X) \rightarrow l_{a}(Y)$ ), that is $\pi(T)$ $=\sup \left\{\sum\left\|T x_{n}\right\|:\left[x_{n}\right] \in l_{w}(E), \varepsilon\left[x_{n}\right] \leqq 1\right\}([7, \S 7] ;[6,2.2] ;$ [4, Anhang 3.5]).

If $T: C_{X}(S) \rightarrow Y$ is absolutely summing, then $T$ has a natural extension, $\hat{T}$, to the space $\mathcal{C}(\mathcal{B}, X)$ of all totally measurable functions [2, II.6.2] defined by $\hat{T} f=\int_{S} f d m, f \in \mathcal{M}(\mathcal{B}, X)$, where $m$ is the representing measure for $T$. Now $\mathcal{C}(\mathcal{B}, X)$ is imbedded in $C_{X}(S)^{\prime \prime}$ since if $f \in \mathcal{M}(\mathcal{B}, X)$ the map $\nu \rightarrow \int_{S} f d \nu$ defines a continuous linear functional on $\operatorname{rcabv}\left(\mathcal{B}, X^{\prime}\right)=C_{X}(S)^{\prime}$ with norm $\|f\|$, and it is easily checked that $\hat{T}$ is just the restriction of $T^{\prime \prime}$ to $M(\mathcal{B}, X)$. Thus if $T$ is absolutely summing, $T^{\prime \prime}$ is absolutely summing [8, Satz 15], and thus $\hat{T}$ is absolutely summing, being the restriction of an absolutely summing map. Note that we must also have $\pi(T)=\pi\left(T^{\prime \prime}\right)=\pi(\hat{T})[8$, Satz 15] since $\hat{T}$ extends $T$ and $T^{\prime \prime}$ extends $\hat{T}$. Also note that we have the following formula for the representing measure

$$
m(B) x=\hat{T}\left(C_{B} x\right), \quad B \in \mathcal{B}, x \in X
$$

Theorem 8. Let $T: C_{X}(S) \rightarrow Y$ be absolutely summing. Then:

(i) The representing measure $m: \mathcal{B} \rightarrow \mathcal{A} S(X, Y)$ has finite variation with respect to the $\pi$-norm (denoted by $\pi-\operatorname{var}(m))$ and $\pi-\operatorname{var}(m) \leqq \pi(T)$.

(ii) The operator $T^{\#}: C(S) \rightarrow A \delta(X, Y)$ is absolutely summing and $\pi\left(T^{\#}\right)$ $\leqq \pi(T)$, where $A \delta(X, Y)$ is supplied with the $\pi$-norm.

Proof. First consider (i). Let $\left\{B_{j}: 1 \leqq j \leqq k\right\}$ be pairwise disjoint from $\mathcal{B}$. Let $\varepsilon>0$ and for each $j$ choose $\sigma_{j} \in F(N)$ and $x_{i j} \in X, i \in \sigma_{j}$, such that $\varepsilon\left[x_{i j}: i \in \sigma_{j}\right] \leqq 1$ and $\sum_{i \in \sigma_{j}}\left\|m\left(B_{j}\right) x_{i j}\right\|+\varepsilon / 2^{j} \geqq \pi\left(m\left(B_{j}\right)\right)$ (Theorem 7 and $[4$, Anhang 3.5]). Thus by formula (2),

$$
\sum_{j=1}^{k} \pi\left(m\left(B_{j}\right)\right) \leqq \varepsilon+\sum_{j=1}^{k} \sum_{i \in \sigma_{j}}\left\|m\left(B_{j}\right) x_{i j}\right\|=\varepsilon+\sum_{j=1}^{k} \sum_{i \in \sigma_{j}}\left\|\hat{T}\left(C_{B_{j}} x_{i j}\right)\right\| .
$$

Now $\hat{T}$ is absolutely summing, and since

$$
\left[C_{B_{j}} x_{i j}: 1 \leqq j \leqq k, i \in \sigma_{j}\right] \in l_{w}(\mathcal{M}(\mathcal{B}, X)),
$$

$$
\begin{aligned}
\sum_{j=1}^{k} \sum_{i \in \sigma_{j}}\left\|\hat{T}\left(C_{B_{j}} x_{i j}\right)\right\| & \\
& \leqq \pi(\hat{T}) \sup \left\{\sum_{j=1}^{k} \sum_{i \in \sigma_{j}}\left|\left\langle\nu, C_{B_{j}} x_{i j}\right\rangle\right|:\|\nu\| \leqq 1, \nu \in \mathcal{M}(\mathcal{B}, X)^{\prime}\right\}
\end{aligned}
$$

$[6,2.2]$. But $\varepsilon\left[x_{i j}: i \in \sigma_{j}\right] \leqq 1$ implies that for any $x^{\prime} \in X^{\prime}$,

$$
\sum_{i \in \sigma_{j}}\left|\left\langle x^{\prime}, x_{i j}\right\rangle\right| \leqq\left\|x^{\prime}\right\| \quad \text { for } 1 \leqq j \leqq k
$$


Thus if $\nu \in \mathcal{M}\left(\mathcal{B}, X^{\prime}\right)[2$, II.9.2, Corollary 1] and $\|\nu\|=v(\nu) \leqq 1$,

(6) $\sum_{j=1}^{k} \sum_{i \in \sigma_{j}}\left|\left\langle\nu, C_{B_{j}} x_{i j}\right\rangle\right|=\sum_{j=1}^{k} \sum_{i \in \sigma_{j}}\left|\left\langle\nu\left(B_{j}\right), x_{i j}\right\rangle\right| \leqq \sum_{j=1}^{k}\left\|\nu\left(B_{j}\right)\right\| \leqq v(\nu)(S) \leqq 1$

by (5). By (3), (4), and (6), we have $\sum_{j=1}^{k} \pi\left(m\left(B_{j}\right)\right) \leqq \varepsilon+\pi(\hat{T})$, and since $\varepsilon$ is arbitrary, $\sum_{j=1}^{k} \pi\left(m\left(B_{j}\right)\right) \leqq \pi(\hat{T})=\pi(T)$. Thus $m: \mathcal{B} \rightarrow \mathcal{A} \delta(X, Y)$ has finite variation with respect to the $\pi$-norm, and $\pi-\operatorname{var}(m) \leqq \pi(T)$.

For (ii), let $\left\{\phi_{j}: 1 \leqq j \leqq k\right\}$ belong to $C(S)$ and be such that $\varepsilon\left[\phi_{j}: 1 \leqq j \leqq k\right]$ $\leqq 1$ in $l_{w}(C(S))$. Let $\varepsilon>0$ and for each $j$ choose $\sigma_{j} \in F(N)$ and $x_{i j} \in X, i \in \sigma_{j}$, such that $\varepsilon\left[x_{i j}: i \in \sigma_{j}\right] \leqq 1$ and

$$
\pi\left(T^{\#} \phi_{j}\right) \leqq \varepsilon / 2^{j}+\sum_{i \in \sigma_{j}}\left\|T^{\#} \phi_{j}\left(x_{i j}\right)\right\| .
$$

Now $\left[\phi_{j} x_{i j}: 1 \leqq j \leqq k, i \in \sigma_{j}\right] \in l_{w}\left(C_{X}(S)\right)$ and since $T$ is absolutely summing,

(8) $\sum_{j=1}^{k} \sum_{i \in \sigma_{j}}\left\|T\left(\phi_{j} x_{i j}\right)\right\| \leqq \pi(T) \sup \left\{\sum_{j=1}^{k} \sum_{i \in \sigma_{j}}\left|\left\langle\nu, \phi_{j} x_{i j}\right\rangle\right|:\|\nu\| \leqq 1, \nu \in C_{X}(S)^{\prime}\right\}$.

But $\varepsilon\left[x_{i j}: i \in \sigma_{j}\right] \leqq 1$ and $\varepsilon\left[\phi_{j}: 1 \leqq j \leqq k\right] \leqq 1$ implies that if $t_{i j}$ are scalars with $\left|t_{i j}\right| \leqq 1$, then

$$
\psi=\sum_{j=1}^{k} \sum_{i \in \sigma_{j}} t_{i j} \phi_{j} x_{i j} \in C_{X}(S)
$$

has norm at most one since

$$
\begin{aligned}
\|\psi\| & =\sup \left\{\left|\left\langle x^{\prime}, \psi(t)\right\rangle\right|:\left\|x^{\prime}\right\| \leqq 1, t \in S\right\} \\
& \leqq \sup \left\{\sum_{j=1}^{k}\left|\phi_{j}(t)\right|: t \in S\right\} \leqq 1 .
\end{aligned}
$$

Thus, if $U=\left\{\nu \in C_{X}(S)^{\prime}:\|\nu\| \leqq 1\right\}$,

$$
\begin{aligned}
\sup \left\{\sum_{j=1}^{k} \sum_{i \in \sigma_{j}}\left|\left\langle\nu, \phi_{j} x_{i j}\right\rangle\right|: \nu \in U\right\} \\
\quad=\sup \{|\langle\nu, \psi\rangle|: \nu \in U, \text { all possible } \psi \text { in }(9)\} \leqq 1 .
\end{aligned}
$$

Using (7), (8), and (10), we obtain $\sum_{j=1}^{k} \pi\left(T^{\#} \phi_{j}\right) \leqq \varepsilon+\pi(T)$ so that $\pi\left(T^{\#}\right)$ $\leqq \pi(T)<\infty$.

Remark. Since the $\pi$-norm dominates the usual operator norm $[6,2.2 .3]$, it follows from Theorem 8 that $m$ has finite variation with respect to the usual operator norm. From [2, III.19.3], it follows that an absolutely summing operator is dominated. This gives an alternate proof of Corollary 5.

We now establish the converse of Theorem 8 and compute the $\pi$-norm of $T$. Again we first establish some preliminary results. 
Lemma 9. Let $Z$ be a dense linear subspace of $X$. Then $l_{s}(Z)$ is dense in $l_{s}(X)$ with respect to the $\varepsilon$-norm.

Proof. Let $\sum x_{n}$ be unconditionally convergent in $X$, and let $\delta>0$. For each $n$ choose $z_{n} \in Z$ such that $\left\|x_{n}-z_{n}\right\|<\delta / 2^{n}$. Then $\sum z_{n}$ is unconditionally convergent and

$$
\varepsilon\left[x_{n}-z_{n}: n \in N\right]=\sup \left\{\sum_{n=1}^{\infty}\left|\left\langle x^{\prime}, x_{n}-z_{n}\right\rangle\right|:\left\|x^{\prime}\right\| \leqq 1, x^{\prime} \in X^{\prime}=Z^{\prime}\right\}<\delta .
$$

(Note that the $\varepsilon$-norm on $l_{s}(Z)$ coincides with the $\varepsilon$-norm from $l_{s}(X)$ since $Z^{\prime}$ and $X^{\prime}$ are isometrically isomorphic.)

Lemma 10. Let $Z$ be a dense linear subspace of $X$. $A$ bounded linear map $T: X \rightarrow Y$ is absolutely summing iff its restriction to $Z, T_{Z}$, is absolutely summable. In this case, $\pi(T)=\pi\left(T_{Z}\right)$.

Proof. If $T$ is absolutely summing, it is clear that $T_{Z}$ is absolutely summing. Assume then that $T_{Z}$ is absolutely summing, and that $\sum x_{n}$ is w.u.c. in $X$. Let $\varepsilon>0$ and for each $n$ pick $z_{n} \in Z$ such that $\left\|x_{n}-z_{n}\right\|<\varepsilon / 2^{n}$. Since $\sum\left|\left\langle x^{\prime}, z_{n}\right\rangle\right|$ $\leqq \sum\left|\left\langle x^{\prime}, x_{n}\right\rangle\right|+\sum\left|\left\langle x^{\prime}, z_{n}-x_{n}\right\rangle\right|$ for each $x^{\prime} \in X^{\prime}=Z^{\prime}, \sum z_{n}$ is w.u.c. in $Z$, and $\sum\left\|T z_{n}\right\|<\infty$. Hence $\sum\left\|T x_{n}\right\|<\infty$, and $T$ is absolutely summing.

The identity $\pi(T)=\pi\left(T_{Z}\right)$ follows from Lemma 9 and the definition of the $\pi$ norm.

Theorem 11. Let $T: C_{X}(S) \rightarrow Y$ be linear and bounded and let $m$ be its representing measure. Then:

(i) If $m(B) \in \mathcal{A} \delta(X, Y)$ for each $B \in \mathcal{B}$ and if $m$ has finite variation with respect to the $\pi$-norm, then $T$ is absolutely summing and $\pi(T) \leqq \pi-\operatorname{var}(m)$.

(ii) If $T^{\#}: C(S) \rightarrow A \delta(X, Y)$ is absolutely summing (with respect to the $\pi$-norm on $A \mathcal{A}(X, Y))$, then $T$ is absolutely summing and $\pi(T) \leqq \pi\left(T^{\#}\right)$.

Proof. First consider (i). It is enough to show that $\hat{T}: \mathcal{M}(\mathcal{B}, X) \rightarrow Y$ is absolutely summing. By Lemma 10 it suffices to show that $\hat{T}$ restricted to $\mathcal{E}(\mathcal{B}, X)$, the dense subspace of $X$-valued step functions [2, II.6.1], is absolutely summing. Let $\left\{f_{j}: 1 \leqq j \leqq k\right\} \subseteq \mathcal{E}(\mathcal{B}, X)$. We may assume that there is a partition $\left\{E_{i}: 1 \leqq i \leqq M\right\}$ of $S$ by nonvoid subsets in $\mathcal{B}$ such that $f_{j}$ $=\sum_{i=1}^{M} C_{E_{i}} x_{i j}, 1 \leqq j \leqq k$. Then $\hat{T} f_{j}=\sum_{i=1}^{M} m\left(E_{i}\right) x_{i j}$, and since each $m\left(E_{i}\right)$ is absolutely summing,

$$
\begin{aligned}
\sum_{j=1}^{k}\left\|\hat{T} f_{j}\right\| & \leqq \sum_{j=1}^{k} \sum_{i=1}^{M}\left\|m\left(E_{i}\right) x_{i j}\right\|=\sum_{i=1}^{M} \sum_{j=1}^{k}\left\|m\left(E_{i}\right) x_{i j}\right\| \\
& \leqq \sum_{i=1}^{M} \pi\left(m\left(E_{i}\right)\right) \sup \left\{\sum_{j=1}^{k}\left|\left\langle x^{\prime}, x_{i j}\right\rangle\right|:\left\|x^{\prime}\right\| \leqq 1\right\}
\end{aligned}
$$

$[6,2.2 .2]$

We now obtain an estimate for each of the terms, $\sup \left\{\sum_{j=1}^{k}\left|\left\langle x^{\prime}, x_{i j}\right\rangle\right|:\left\|x^{\prime}\right\|\right.$ 
$\leqq 1\}$, on the right-hand side of (11). Fix $i \in\{1, \ldots, M\}$ and pick $s_{i} \in E_{i}$. Let $x^{\prime} \in X^{\prime}$ be such that $\left\|x^{\prime}\right\| \leqq 1$ and define $\nu \in \mathcal{E}(\mathcal{B}, X)^{\prime}$ by $\langle\nu, f\rangle=\left\langle x^{\prime}, f\left(s_{i}\right)\right\rangle$, $f \in \mathcal{E}(\mathcal{B}, X)$. Note $\|\nu\|=1$ and $\left\langle\nu, f_{j}\right\rangle=x_{i j}$. Thus

$$
\sum_{j=1}^{k}\left|\left\langle x^{\prime}, x_{i j}\right\rangle\right|=\sum_{j=1}^{k}\left|\left\langle\nu, f_{j}\right\rangle\right| \leqq \sup \left\{\sum_{j=1}^{k}\left|\left\langle\mu, f_{j}\right\rangle\right|:\|\mu\| \leqq 1, \mu \in \mathcal{E}(\mathcal{B}, X)^{\prime}\right\}
$$

so that, for each $i \in\{1, \ldots, M\}$,

$$
\begin{aligned}
\sup \left\{\sum_{j=1}^{k}\left|\left\langle x^{\prime}, x_{i j}\right\rangle\right|:\left\|x^{\prime}\right\| \leqq 1\right\} & \\
& \leqq \sup \left\{\sum_{j=1}^{k}\left|\left\langle\mu, f_{j}\right\rangle\right|:\|\mu\| \leqq 1, \mu \in \mathcal{E}(\mathcal{B}, X)^{\prime}\right\} .
\end{aligned}
$$

From (11) and (12), we have

$$
\begin{aligned}
\sum_{j=1}^{k}\left\|\hat{T} f_{j}\right\| & \leqq \sum_{i=1}^{M} \pi\left(m\left(E_{i}\right)\right) \sup \left\{\sum_{j=1}^{k}\left|\left\langle\mu, f_{j}\right\rangle\right|:\|\mu\| \leqq 1, \mu \in \mathcal{E}(\mathcal{B}, X)^{\prime}\right\} \\
& \leqq \pi-\operatorname{var}(m) \sup \left\{\sum_{j=1}^{k}\left|\left\langle\mu, f_{j}\right\rangle\right|:\|\mu\| \leqq 1, \mu \in \mathcal{E}(\mathcal{B}, X)^{\prime}\right\} .
\end{aligned}
$$

It now follows from (13) and 2.2.2 of [6] that $\hat{T}$ is absolutely summing and $\pi(\hat{T})=\pi(T) \leqq \pi-\operatorname{var}(m)$.

For (ii) note that if $f_{1}, \ldots, f_{n}$ is a finite family of functions from $C_{X}(S)$ and $\varepsilon>0$, there is a finite family $\left\{\phi_{j}: j \in \sigma\right\}$ in $C(S)$ and $\left\{x_{i j}: 1 \leqq i \leqq n, j \in \sigma\right\}$ such that $0 \leqq \phi_{j} \leqq 1, \sum_{j \in \sigma} \phi_{j}=1$, and $\left\|f_{i}-\sum_{j \in \sigma} \phi_{j} x_{i j}\right\|<\varepsilon$ for $i=1, \ldots, n$. (See the proof of Proposition 2 of III.19.1 of [2].) The method of proof in part (i) can now be applied.

Combining Theorems 8 and 11, we obtain

Theorem 12. Let $T: C_{X}(S)$ be linear and bounded with representing measure $m$. The following three conditions are equivalent:

(i) $T$ is absolutely summing,

(ii) Each $m(B) \in \mathcal{A} \delta(X, Y), B \in \mathcal{B}$, and $m$ has finite $\pi$-variation.

(iii) $T^{\#}: C(S) \rightarrow \mathcal{A} \delta(X, Y)$ is absolutely summing with respect to the $\pi$-norm on As $(X, Y)$.

When (i), (ii) or (iii) holds, $\pi(T)=\pi-\operatorname{var}(m)=\pi\left(T^{\#}\right)$.

Since the absolutely summing norm dominates the usual operator norm if $m: \mathcal{B} \rightarrow \mathcal{A} \delta(X, Y)$ has finite variation with respect to $\pi$ (as in Theorem 12(ii)), then $m$ has finite variation with respect to the operator norm. The converse of this statement is false. We now give an example showing this. The example and Theorem 13 are both due to the referee.

Theorem 13. Let $X$ and $Y$ be $B$-spaces such that if $m: B \rightarrow A S(X, Y)$ has finite variation with respect to the usual operator norm, then $m$ has finite variation with 
respect to the absolutely summing norm. If $S$ is infinite, then there is a constant $\rho$ such that

$$
\pi(T) \leqq \rho\|T\| \quad \text { for all } T \in \mathcal{A} \delta(X, Y)
$$

Proof. Assume the constant $\rho$ does not exist. Then there is a sequence $T_{n} \in A \delta(X, Y)$ such that $\pi\left(T_{n}\right) \geqq 1$ and $\left\|T_{n}\right\| \leqq 1 / 2^{n}$. Let $\left\{s_{n}\right\}$ be a sequence of distinct points in $S$. Then the measure $m=\sum T_{n} \delta_{s_{n}}$, where $\delta_{s_{n}}$ is the Dirac measure concentrated at $s_{n}$, has finite \|\| -variation but infinite $\pi$-variation.

Example 1. Let $X=Y=C_{0}$. Then condition (14) does not hold since if $T_{n}\left(t_{1}, \ldots, t_{n}, t_{n+1}, \ldots\right)=\left(t_{1}, \ldots, t_{n}, 0,0, \ldots\right)$, then $\left\|T_{n}\right\|=1$ and $\pi\left(T_{n}\right)=n$.

\section{REFERENCES}

1. C. Bessaga and A. Pecłzyński, On bases and unconditional convergence of series in Banach spaces, Studia Math. 17 (1958), 151-164. MR 22 \#5872.

2. N. Dinculeanu, Vector measures, Internat. Series of Monographs in Pure and Appl. Math., vol. 95, Pergamon Press, Oxford; VEB Deutscher Verlag der Wissenschaften, Berlin, 1967. MR 34 \# $6011 \mathrm{~b}$.

3. N. Dunford and J. T. Schwartz, Linear operators. I: General theory, Pure and Appl. Math., vol. 7, Interscience, New York, 1958. MR 22 \#8302.

4. K. Floret and J. Wloka, Einführung in die Theorie der lokalkonvexen Räume, Lecture Notes in Math., no. 56, Springer-Verlag, Berlin and New York, 1968. MR 37 \# 1945.

5. C. Foiaş and I. Singer, Some remarks on the representation of linear operators in spaces of vectorvalued continuous functions, Rev. Roumaine Math. Pures Appl. 5 (1960), 729-752. MR 24 \# A1618.

6. A. Pietsch, Nukleare lokalkonvexe Räume, Schriftenreihe der Institute für Mathematik bei der Deutschen Akademie der Wissenschaften zu Berlin. Reihe A, Reine Mathematik, Heft 1, AkademieVerlag, Berlin, 1965. MR 31 \#6114.

7.— Absolut summierende Abbildungen in lokalkonvexen Räumen, Math. Nachr. 27 (1963), 77-103. MR 28 \# 1473.

8.—, Quasinukleare Abbildungen in normierten Räumen, Math. Ann. 165 (1966), 76-90. MR 33 \#6412.

9.—, Absolut p-summierende Abbildungen in normrerten Räumen, Studia Math. 28 (1966/67), 333-353. MR 35 \# 7162.

Department of Mathematics, New Mexico State University, Las Cruces, New Mexico 88001 